\title{
M-LEARNING IN EDUCATION
}

\author{
P.Amuthabala ${ }^{1}$, Annapurna.P ${ }^{2}$, Vanishree. $M^{3}$, Lavanya. ${ }^{4}$ \\ ${ }^{1}$ Senior Lecturer, Information Science and Engineering, Atria Institute of technology, Karnataka, India \\ ${ }^{2}$ Student, Information Science and Engineering, Atria Institute of technology, Karnataka, India \\ ${ }^{3}$ Student, Information Science and Engineering, Atria Institute of technology, Karnataka, India \\ ${ }^{4}$ Student, Information Science and Engineering, Atria Institute of technology, Karnataka, India
}

\begin{abstract}
Computers are still lab and class oriented but now as tablets and mobiles have an advanced technology, which is advancing day by day. Computers can be used for learning beyond classroom through mobile devices which leads to a more practical and logical learning. This is based on group learning which takes place through a mobile device. Most of the mobiles are based on android technology and support collaborative learning. Collaborative learning using mobile devices can make users to learn the particular subject or language through their mobile phones they can take test and result will be displayed after the test held, the notes and the details of the user will be stored in the database where space will be taken in the cloud which is used to store the data, it focus on collaborative learning and peer-review learning both are related concepts where they can share education resources and information and feedback can be taken by the students and result will be displayed. Client server system is used to provide for all these feature.
\end{abstract}

Keywords: Mobile devices, qr-codes, peer-reviewed learning, educational resources, PDA's(personal digital assistant), wireless programming, encryption, Android Cloud to Device Messaging Framework, Cloud to device messaging server, SDK, JDK, JVM, JPDA.

\section{INTRODUCTION}

The learning technology which is used here is a group learning method which takes place through a mobile device. It makes use of wireless communication technology. Here students can grasp the concepts and learn the concepts through mobile device, by interacting with each other and also with the professors, and for this android technology can be used.

According to Thomas [2], data is conceptualized in such learning and follows miniaturization and convenience of portable computing learners follow content and context in an authentic learning environment rather than simple transmission of context in classroom can be carried out through qr-codes, augmented reality and connecting multiple context.

Cloud used with android is very helpful as an application developed using android can be launched in cloud and these apps and services can be accessed using cloud computation. Cloud supports on demand computing i.e. users can access supercomputing level using other access point like i phone, BB etc whenever needed. This computing is discussed [7], Alexander. Client server module is used here the cloud providers create application interface so that software developers create client application to access various functions.
Collaborative learning assistance is a collaborative usage and frame work where the users can access and learn the course studies anywhere and at any location as compared to the classroom and lab studies which are carried out at a given time which depends on the availability of respective faculties and also a schedule given by the university has to be followed which is restrictive and time bound. So, the learning can be more extensive and interactive using learning assistance the user whenever free can access the learning assistant and reach the faculty through notes and chats, media files can also be exchanged among the students and faculties they can access the faculties according to the course irrespective of time and location. It aims at:

- Collaborative learning.

- Organizing resources and activities.

- Peer-reviewed learning.

- Auto evaluation.

- $\quad$ Sharing educational resources $\mathrm{t} 8 \mathrm{vdx} 9$

\section{COLLABORATIVE LEARNING}

Collaborative learning through android application using mobile devices is developed in such a way that student who all are interested can learn particular subject by enrolling themselves into the application. Where faculties are also enrolled to the particular department staffs can upload the files in the database form where students can download and learn. 
Student can discuss among themselves through chat option where group and individual chat can be done this can be viewed by staff also and all these data will be stored in the cloud and user can retrieve it, cloud has to provide services for user to retrieve the data from the database which is stored in cloud.

\section{ARCHITECTURE DIAGRAM}

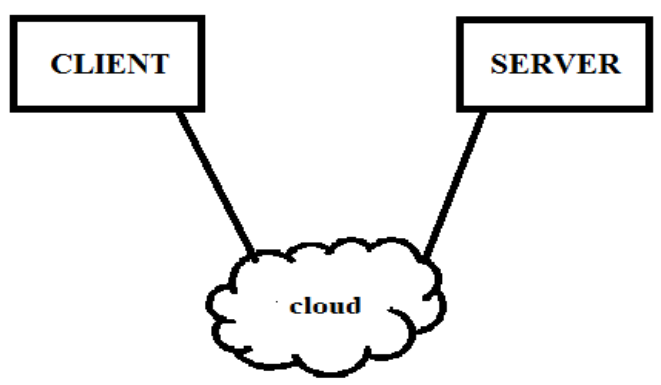

Fig-1: Client-cloud-Server

\subsection{Client}

Clients will be student and staff where students staffs needs to enroll in the application where administrator have authority of adding students and staff and also deleting all the clients information is stored under cloud here using godadi cloud space to store the data and the clients information. They can access cloud through protocol and students can download the data from the database staff can upload the files to the database where students can retrieve data from the database.

\subsection{Server}

Server refer to the admin, admin can add both students and staffs and the department as well, and delete them if it is not needed all these authorities is only for the server this will convey request from the client to the server which is stored in the cloud, admin have direct access to the database. It involves the server's database system which allows centralized client server architecture.

Larger files, Google blob store is used supports for the larger files, use app-engine server to store large loaded data for reducing server load. In future implementation it can also consider cloud SQL by Google as addition storage option.

\subsection{HTTP Protocol}

The server sends the response for the user, who made request to the particular file which has saved in the database this activity to exist protocol is used which is called hypertext transfer protocol where the file can be transferred from the database or to open particular page in the site need access this job done by hypertext transfer protocol, the centralized database's use to store data and receiving of information from the server(database), as multiple clients request same or different resources the server has to provide response for the requested user based on the priority, for very high load of data to transfer use clusters [12] where different nodes are connected in network to store data, used in this education app.

For handling huge data application uses multiple purposes for downloads and uploads these are handled by the server.

\section{EXISTING SYSTEM}

Very few mobile learning languages app are designed from 2001. Stanford learning lab provides development in mobile language learning by using personal digital assistant such that they are very simple applications like vocabulary learning and simple quiz held in text format.

Though it is designed for simple learning methods through mobiles, it covers simple methods app like text based learning and quiz the next is to improve foe the higher level of learning including complex language and other levels of learning where students may learn different techniques and knowledge without attending classes and lab conducted in normal education field.

\section{PROPOSED SYSTEM}

Advance in mobile learning techniques (m-learning) led to develop an application for education through mobiles in very diverse range of fields, here it is designed an application where the students can learn a particular subject in the database where the admin provided and staff can upload the material in the database this material can be downloaded by the students and they can learn from it the downloaded data will be saved in the file location mentioned before the test can be conducted and result displayed for the test, wide range of changes had made compared to the before application and the cloud is used to store the data in the server. It has been argued that new mobile technologies allow for learning programs to be developed by comparison to traditional learning are more secured, learner-centered, situated, collaborative, and lifelong. An additional advantage often overlooked particularly for mobile phones is that there is a natural existence for phones to speak into and listen from (i.e. audio interaction). Applications that involve speaking and listening may actually be better suited to mobile phones than the PC environment.

\section{LITERATURE SURVEY}

As discussed Alexander [7] is a combination of connections and servers. It allows users to run and host web applications on Google infrastructure. The application environment supports dynamic web serving. It is also supports storage querying, sorting and transferring of data and authentication user this makes it easy to use and free to get started. 


\section{Case Study}

According to, L Dyson,, E. Lawrence [4] case studies were conducted in a university.

\subsection{Case Study 1:}

Field work which was supported by a mobile device for 1 st year, 1st semester subject for IT \& business studies 340 students in 1 st semester.

$1^{\text {st }}$ Trial: Personal digital assistant given to each student team. Problem: They faced the problem of poor usability the distribution of personal digital assistant needed to be tracked to ensure they were not stolen. Cost of personal digital assistant was an overhead expense.

$2^{\text {nd }}$ Trial: Students used their own devices.

Problem: When student were given their own devices number of students could not be accounted and students were not sure about data to collect and use over developer or download or share data.

Final: Instructions about data and usage were given then securing the data i.e. photos, record taken was a problem.

\subsection{Case Study 2:}

Here operation and mobile interaction is discussed, where the student $\mathrm{A}$ is activates discussion through a doubt or a query, this is viewed by another student B who adds his/her view points. The $\mathrm{C}$ student collects responses and results of interaction are displayed to B. Where it is their choice to interact. And professor can also access the student doubt or a query and he can respond to the student requests by uploading the data in the database.

\section{Case Study 3:}

Here mobile technology is used to learn, this study done on students at the end of 1 st semester and students use personal digital assistant, 25 student are enrolled in a subject and 11 use personal digital assistant among them. So the conclusion taken from the students was as follows:

Out of 11, 9 students supported that personal digital assistant were better for wireless programming as they found it easy to interact and carryout the program logically.

Concluding about the use of personal digital assistant theoretical understanding then out of 11, 6 agreed for the use of personal digital assistant in theoretical learning as they understood theoretical concepts better through mobile devices. The last survey was done on the issue whether personal digital assistant were really use full outside the classroom for which 8 students out of 11 agreed that personal digital assistant were helpful even outside the premises of location and time.

\section{SECURE CONNECTIVITY}

As security was a main issue as students could access mails and media transfer was also supported to secure transmission of data need of encryption so according to ref[10] William J, encryption method is used on the client side and similarly decryption was used at server side. There were many options, included in this to secure the data.

Advance encryption standard was also used to secure large media files for transmission across client server to avoid unwanted access and unwanted modification of data.

Use of encryption added the advantage of optimization of data even if bulk data needed to be encrypted and sent it would not hamper the performance.

And this generated a high throughput and how response latency to enhance and segregate the load a 3rd party [8] (Android Cloud to Device Messaging Framework) app server is used to send height weight message to android app and this app uses an existing connection of Google.

The architecture here consisted of the following components:

- Mobile device

- 3 rd party application server

- Cloud to device messaging server

Which also included storage and the load was not only on one server but distributed.

The credentials used in this architecture was:

- $\quad$ Sender ID

- $\quad$ Application ID

- Registration ID

- Google user A/C

- Sender account token

\section{CLUSTER GRID}

The "collaborator" which is a research base with multiple users for high performance computing. This was designed as communication path $\mathrm{b} / \mathrm{w}$ universities using national $\mathrm{n} / \mathrm{w}$ infrastructure for education and research. This gave million of a cluster.

Cluster responsibility changing as new machines evolve and are heterogeneous. 4 platforms are available on cluster:

- Intel X 86 Linux

- Intel X 86-64 Linux

- $\quad$ Sun SPARC Solaris

- $\quad$ Sun Intel X 86-64 Solaris

The processors available in cluster are:

- Intel xeon Processor- Ave quickest

- Advance Micro Device with real world workloads 
- $\quad$ IBM cell broadband engine:

- Singles and double precision floating point.

\section{EXPERIMENTAL RESULT}

Existing System

The existing system uses android software development kit where all the levels of API have to be updated but uses the 19th level of API.

1. Install the Software Development Kit starter package, both windows and mac have different packages.

2. Install the Abstract Data Type Plug in for Eclipse.

3. Add Android platforms and other components to Software Development Kit.

Download Java Development Tool kit: The Java Development Tools project provides the tool plug-ins that implements a Java Integrated Drive Electronics supporting the development of any Java application includes Eclipse plugins. It adds a Java project nature and Java perspective to the Eclipse Workbench, views, editors, and code merging. The Java Development Tools project allows Eclipse to be a development environment for itself. Features include:

- Java projects with source files which is arranged in packages

- Editing with keyword and syntax coloring, outline showing declaration structure

- Search

- Compare

- Compile JCK compliant Java compiler

- Run Java programs in a separate JVM

- Debug programs with JPDA

- Create an Android Emulator Device

The Android tools include an emulator, the emulator similar to the android page which displayed in mobile devices, in most cases and allows testing application without having a real device. Emulate one or several devices with different configurations where each configuration is defined via an "Android Virtual Device".

Proposed System works as follows:

Input

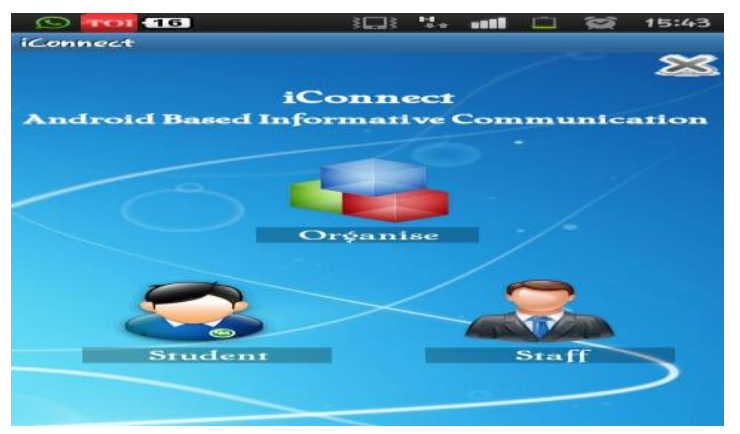

Fig-2:Staff-Organise-Student
Organise $\rightarrow$ Department (add and remove)

Students (add and remove)

Staffs (add and remove)

Password (change)

Students $\rightarrow$ Notes (can be downloaded)

Online test (can be taken)

Chat (group or individual)

Password (change)

Staff $\rightarrow$ Blog (can upload the file)

Notes (can be downloaded)

Chat (group or individual)

Password (change)

\section{Output}

Online test (taken by the students)

Chat (individual chats or group chats which includes students and faculty from any location)

\section{Software Requirements}

$>$ Operating System : Android

$>$ Package : Java Development Kit 1.5 and above

$>$ Software : Eclipse Juno

$>$ Language : ANDROID SDK

$>$ Documentation : Ms-Office

\section{Hardware Requirements}

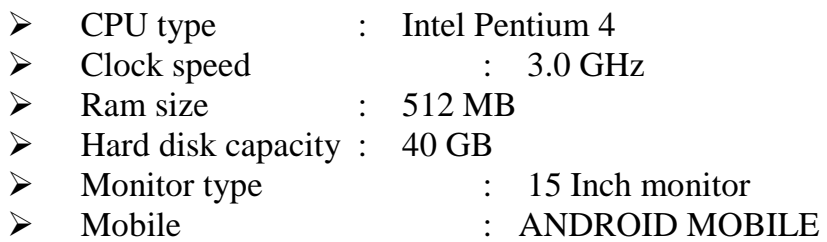

\section{FUTURE ENHANCEMENT}

The learning is objective which is not always supportive. New teaching approaches can be added project based learning can be initiated here.

A new system can be developed where lectures uses the system to engage student material throughout a lecturer, and even students can upload their own notes and questions to entire class available.

Cloud space can enhance so that more data can be stored in the system, more than one subject can be added in each of the department, and that data can be stored in cloud and media transmission be included like video chat among the students and also staff can clear student's doubts so far txt message's among students and staff is designed if cloud space is more it can store many message's and data. 


\section{CONCLUSIONS}

Cloud computing is evolving and advancing it is also having an advantage in terms of cost efficiency and environment. It allows to occupy space for storing database which includes student and staff personal information and the course details and other course related data. Where it provide services for accepting or delivering data from database to the particular user

Collaborative learning ability of making judgment and problem solving. Students here immediately get to know about the correctness of the answer, which is very useful and time managing. Here online transaction processing systems are implemented to improve the speed of performance which is increased in hardware devices.

\section{REFERENCES}

[1]. Acharya, S., \& Teltscher, S. "ITU estimates two billion people online by end 2010", Newsroom: Press Release Retrieved 11 November, 2012, from:

http://www.itu.int/net/pressoffice/press_releases/2010/39.aspx

[2]. Cochrane, Thomas. "mLearning: why? What? Where? How?." Proceedings of the 28th ASCILITE Conference, ASCILITE. 2011.

[3]. Naismith L., Lonsdale P., Vavoula G., and Sharples M. Literature review in mobile technologies and learning, 2004.

[4]. L. Dyson, A. Litcheld, E. Lawrence, R. Raban, and P. Leijdekkers. Advancing the m-learning research agenda for active, experiential learning, 2007.

[5]. Cook J. Phases of mobile learning. joint european summer school on technology enhanced learning, May 2009.

[6]. Barker, Andrea, Greig Krull, and Brenda Mallinson. "A proposed theoretical model for m-learning adoption in developing countries." Proceedings of mLearn. Vol. 2005.

[7]. Zahariev, Alexander. "Google app engine" TKK T110.5190 Seminar on Internetworking. 2009.

[8]. "Android Cloud to Device Messaging Framework" Available online

at:

https://developers.google.com/android/c2dm/

[9]. "Google Cloud Messaging for Android" Available online at:http://developer.android.com/google/gcm/index.html

[10]. Bolosky, William J., et al. "Paxos replicated state machines as the basis of a high-performance data store." Symposium on Networked Systems Design and Implementation (NSDI). 2011.

[11]. Sultan, Nabil. "Cloud computing for education: A new dawn?." International Journal of Information Management 30.2 (2010): 109-116.

[12]. The NCIT Cluster Resources User's Guide, University Politehnica of Bucharest Cluster, Retrieved 21.12.2012, Available online:

https://cluster.grid.pub.ro/index.php/component/docman/doc_ download/2-cluster-guide-v3-1-short-version

\section{BIOGRAPHIES}

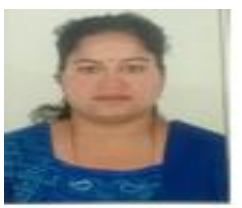

P. Amuthabala has obtained her B.E in Computer science Engineering at Avanishilingam University in Coimbatore, Tamilnadu, India in 2002 and her M.E degree in Software Engineering at Bangalore University in Bangalore, Karnataka, India in 2011.She is working as a Senior Lecturer in Information Science Department at Atria Institute of Technology, Bangalore, Karnataka. Her research areas of Interest include Data Mining Data warehousing and Cloud Computing.

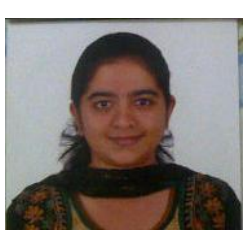

Annapurna.P Has obtained Diploma in computer science at BTE (Board of Technical Education) Bangalore, Karnataka in 2011, Doing B.E in Information Science Engineering at Visvesvaraya Technology University, Atria Institute Of Technology, Bangalore.

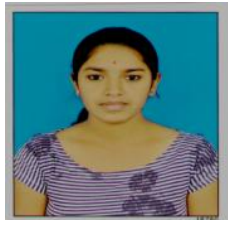

Bangalore.

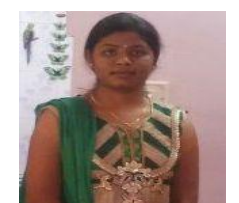

Vanishree.M Has obtained Diploma in computer science at BTE (Board of Technical Education) Bangalore, Karnataka in 2011, Doing B.E in Information Science Engineering at Visvesvaraya Technology University, Atria Institute Of Technology,

Lavanya.D Doing B.E in Information Science Engineering at Visvesvaraya Technology University, Atria Institute Of Technology, Bangalore. 